\title{
Clinical Impact of Pneumothorax in Patients With Pneumocystis Jirovecii Pneumonia and Respiratory Failure in An HIV-Negative Cohort
}

\section{Ji Soo Choi}

Yonsei university College of Medicine

\section{Se Hyun Kwak}

Yonsei university College of Medicine

Min Chul Kim

Yonsei university College of Medicine

Chang Hwan Seol

Yonsei university College of Medicine

\section{Sung Ryeol Kim}

Yonsei university College of Medicine

\section{Byung Hoon Park}

Yonsei university College of Medicine

\section{Eun Hye Lee}

Yonsei university College of Medicine

\section{Seung Hyun Yong}

Yonsei University College of Medicine

Ah Young Leem

Yonsei University College of Medicine

\section{Song Yee Kim}

Yonsei University College of Medicine

\section{Sang Hoon Lee}

Yonsei University College of Medicine

Kyungsoo Chung

Yonsei University College of Medicine

\section{Eun Young Kim}

Yonsei University College of Medicine

Ji Ye Jung

Yonsei University College of Medicine

\section{Young Ae Kang}

Yonsei University College of Medicine

Moo Suk Park 
Yonsei University College of Medicine

\section{Young Sam Kim}

Yonsei University College of Medicine

Su Hwan Lee ( $\nabla$ hihogogo@yuhs.ac)

Yonsei University College of Medicine

\section{Research Article}

Keywords: Pneumonia, Pneumocystis, Respiratory Insufficiency, Pneumothorax, Prognosis, Risk Factors

Posted Date: October 1st, 2021

DOl: https://doi.org/10.21203/rs.3.rs-861380/v2

License: (c) (1) This work is licensed under a Creative Commons Attribution 4.0 International License. Read Full License

Version of Record: A version of this preprint was published at BMC Pulmonary Medicine on January 8th, 2022. See the published version at https://doi.org/10.1186/s12890-021-01812-z. 


\section{Abstract}

Background: Pneumocystis jirovecii pneumonia (PCP) with acute respiratory failure can result in development of pneumothorax during treatment. This study aimed to identify the incidence and related factors of pneumothorax in patients with PCP and acute respiratory failure and to analyze their prognosis.

Methods: We retrospectively reviewed the occurrence of pneumothorax, including clinical characteristics and results of other examinations, in 119 non-human immunodeficiency virus patients with PCP and respiratory failure requiring mechanical ventilator treatment in a medical intensive care unit (ICU) at a tertiary-care center between July 2016 and April 2019.

Results: The 28-day survival rate was $62.2 \%(\mathrm{~N}=74)$. Twenty-two patients (18.5\%) developed pneumothorax during ventilator treatment, with $45(37.8 \%)$ eventually requiring a tracheostomy due to weaning failure. Cytomegalovirus co-infection (odds ratio 13.9; $p=0.013$ ) was related with occurrence of pneumothorax. Of 74 patients with 28-day survival data, 15 (20.3\%) developed pneumothorax. In those patients, five survived $(p=0.048)$ and only two were successfully weaned from mechanical ventilation $(p=0.037)$.

Conclusions: Patients with PCP and acute respiratory failure who developed pneumothorax did not have increased 28-day mortality, however pneumothorax increased in patient with cytomegalovirus coinfection, pneumothorax might have difficulty in and prolonged weaning from mechanical ventilators, which clinicians should be aware of when planning treatment for such patients.

\section{Background}

The incidence of Pneumocystis jirovecii pneumonia (PCP) in patients without human immunodeficiency virus (HIV) has increased as more patients receive chemotherapy or immunosuppressive agents [1]. The disease progress in these patients is rapid, and the prognosis is worse compared to that of PCP patients with HIV [2]. Furthermore, when complicated by respiratory failure, the prognosis is poor and mortality rate is high [3].

Pneumothorax is one of the complications of PCP [4]. The prevalence of pneumothorax ranged from 13$61 \%$ in PCP patients with and without HIV in previous study [5]. There are several studies on the association between the occurrence of pneumothorax in PCP and prognosis in patients with HIV [6-8]. In one study of patients without HIV, development of pneumothorax was related to poor prognosis, including high acutely physiology and chronic health evaluation (APACHE) III scores, prolonged positive pressure ventilation, and intubation delay, in patients with acute respiratory failure complicating PCP [9]. However, the relationship between pneumothorax and prognosis of PCP with acute respiratory failure in patients without HIV remains unclear [5]. 
The aim of this study was to identify the incidence of pneumothorax in patients with PCP and acute respiratory failure without HIV and to further analyze related factors. In addition, we investigated the prognosis, including success rates of ventilator weaning, of these patients and progression of disease.

\section{Methods}

\section{Study population}

In this study, we retrospectively investigated the medical records of 1,210 patients who were admitted to a medical intensive care unit at a tertiary care university hospital in South Korea between July 2016 and April 2019. Patients with PCP without HIV who needed mechanical ventilation due to respiratory failure in ICU care were included. Three criteria were applied for the diagnosis of PCP: (1) immunocompromised status from chemotherapy, immunosuppressant usage, or long term use of steroid with clinical symptoms of pneumonia, such as cough, sputum, fever, and dyspnea; (2) P. jirovecii DNA must be confirmed using polymerase chain reaction (PCR) assays from patient sputum samples, endotracheal aspirates, or bronchoalveolar lavage fluids; and (3) radiologic finding from chest computed tomography (CT) representing typical patterns of PCP, including bilateral interstitial opacities, ground glass opacities, or septal thickening must be evident. $P$. jirovecii PCR-positive patients who did not receive treatment for PCP were excluded due to possible false positivity.

\section{Data collection}

Patient baseline characteristics, laboratory findings, and information on disease severity, such as APACHE II score, Sequential Organ Failure Assessment (SOFA) score, and Simplified Acute Physiology Score (SAPS) II, were collected from data obtained within 24 hours of ICU admission. We reviewed the mechanical ventilator $(\mathrm{MV})$ parameters, including respiratory rate, tidal volume $(\mathrm{mL} /$ predicted body weight), peak inspiratory pressure, positive expiratory end pressure (PEEP), dynamic driving pressure (the difference between peak inspiratory pressure and PEEP) within 24 hours after MV initiation. We also reviewed peak inspiratory pressure three days after- and maximal peak pressure within a week after MV initiation. Concomitant Cytomegalovirus (CMV) antigenemia was decided through a quantitative PCR test. The cutoff value for clinically meaningful positive CMV PCR result was higher or equal 1500 copies/mL [10].

Occurrence of pneumothorax was evaluated via radiologic manifestations with chest radiography and $\mathrm{CT}$. We investigated the prognosis of patients, including successful MV weaning outcomes, ICU length of stay, 28-day mortality and in-hospital mortality. Approval for this study was provided by the institutional review board of Yongin Severance Hospital (IRB 9-2021-0045). The need for informed consent was waived due to the retrospective nature of this study. This study was conducted in accordance with the tenets set by the Declaration of Helsinki.

\section{Management of PCP}


All study patients were administered intravenous or oral trimethoprim (15-20 mg/kg per day) and sulfamethoxazole (75-100 mg/kg per day) as first-line treatment for PCP. The duration of planned treatment was three weeks. We changed to second-line treatment using primaquine $(15-30 \mathrm{mg} /$ day) and clindamycin ( $1800 \mathrm{mg} /$ day) or pentamidine $(4 \mathrm{mg} / \mathrm{kg}$ per day) in patients who did not present clinical improvement. Twenty-one (17.6\%) patients were treated with second-line medication due to no clinical improvement or side effects of the first-line medication. In addition, all patients were administered adjuvant corticosteroid ( $40 \mathrm{mg}$ prednisolone twice daily for five days, followed by $40 \mathrm{mg}$ prednisolone twice daily for five days, after which $20 \mathrm{mg}$ prednisolone twice daily for 11 days was given). Patients requiring $\mathrm{MV}$ treatment for more than two weeks due to $\mathrm{MV}$ weaning failure underwent tracheostomy.

\section{Statistical Analysis}

Categorical variables were presented as frequencies and percentages with comparisons done using a chisquare test. Continuous variables were presented as means and standard deviations if the distribution was normal, and as interquartile ranges (IQRs) if the distribution was not normal. For comparisons of continuous variables, a Student's t-test and Mann-Whitney $U$ test were used. A multivariable logistic regression analysis was performed with pre-specified covariates. Odds ratios (ORs) and $95 \%$ confidence intervals (Cls) were also calculated. A $p$-value $<0.05$ was considered significant for all analysis. All data were analyzed statistically using IBM SPSS version 25.0 software (IBM Corp., Armonk, NY, USA).

\section{Results}

\section{Study flow}

A total of 160 patients with clinically confirmed PCP and on treatment for it were enrolled. Forty one patients who had respiratory failure, but had not received MV treatment and had undergone tracheostomy before ICU admission were excluded; therefore, 119 patients were finally included in our study (Fig. 1).

\section{Baseline characteristics of the study participants}

The baseline characteristics of study patients are summarized in Table 1. Among the 119 patients reviewed, 76 (63.9\%) were male and 43 (36.1\%) were female. Median age was 65.0 (IQR, 56.0-72.0) years. Median APACHE II score was 26.5 (IQR, 20.0-32.0). Thirty-four patients (28.6\%) were smokers, and $31(26.1 \%)$ had underlying chronic lung diseases, including airway disease $(n=9,7.3 \%)$ and interstitial lung disease $(n=22,18.5 \%)$. The main cause of immunodeficiency was a solid cancer $(n=37,31.1 \%)$, followed by immunosuppressive agent use $(n=27,22.7 \%)$, and hematologic malignancy $(n=22,18.5 \%)$. Forty-one patients (34.5\%) needed continuous renal replacement therapy (CRRT) because of acute kidney injury. Concomitant $\mathrm{CMV}$ antigenemia was noted in 75 patients $(63.0 \%)$. 
Table 1

Baseline characteristics of patients with Pneumocystis jirovecci pneumonia requiring mechanical ventilation in intensive care unit

\begin{tabular}{|c|c|}
\hline Patient characteristics & $N=119$ \\
\hline Median age, years & $65.0(56.0-72.0)$ \\
\hline Sex (male) & $76(63.9)$ \\
\hline Height, cm & $163.0(156.0-170.0)$ \\
\hline $\mathrm{BMI}, \mathrm{kg} / \mathrm{m}^{2}$ & $22.3(20.1-25.0)$ \\
\hline Ever smoker & $34(28.6)$ \\
\hline Charlson Comorbidity Index & $3(2-4)$ \\
\hline \multicolumn{2}{|l|}{ Comorbidity disease } \\
\hline Congestive heart failure & $11(9.2)$ \\
\hline Coronary arterial disease & $21(17.6)$ \\
\hline Chronic lung disease - airway & $9(7.6)$ \\
\hline Chronic lung disease - ILD & $22(18.5)$ \\
\hline Chronic kidney disease & $31(26.1)$ \\
\hline Chronic liver disease & $9(7.6)$ \\
\hline Cerebrovascular disease & $4(3.4)$ \\
\hline Solid cancer & $37(31.1)$ \\
\hline Hematologic malignancy & $22(18.5)$ \\
\hline Immunosuppressive agent use & $27(22.7)$ \\
\hline CRRT due to AKI & $41(34.5)$ \\
\hline APACHE II score & $26.5(20.0-32.0)$ \\
\hline SOFA score & $8(6-11)$ \\
\hline SAPS II & $37.0(29.0-18.8)$ \\
\hline Cytomegalovirus antigenemia & $75(63.0)$ \\
\hline \multicolumn{2}{|l|}{ Clinical parameters } \\
\hline \multicolumn{2}{|c|}{ Data are presented by numbers (\%) or median (IQR) unless indicated otherwise. } \\
\hline \multicolumn{2}{|c|}{$\begin{array}{l}\text { IQR, interquartile range; BMI, body mass index; ILD, interstitial lung disease; CRRT, continuous renal } \\
\text { replacement therapy; AKI, acute kidney injury; APACHE II, Acute Physiology and Chronic Health } \\
\text { Evaluation II; SOFA, Sequential Organ Failure Assessment; SAPS II, simplified acute physiology score } \\
\text { II; WBC, white blood cell; BUN, blood urea nitrogen }\end{array}$} \\
\hline
\end{tabular}




\begin{tabular}{|c|c|}
\hline Patient characteristics & $N=119$ \\
\hline WBC, $\times 10^{3} / \mu \mathrm{L}$ & $10.2(5.9-15.3)$ \\
\hline Platelet, $\times 10^{3} / \mu \mathrm{L}$ & $132.5(60.8-246.0)$ \\
\hline $\mathrm{BUN}, \mathrm{mg} / \mathrm{dL}$ & $29.5(19.5-44.6)$ \\
\hline Creatinine, $\mathrm{mg} / \mathrm{dL}$ & $1.07(0.62-1.90)$ \\
\hline Albumin, g/dL & $2.5(2.2-2.7)$ \\
\hline Lactate, $\mathrm{mmol} / \mathrm{L}$ & $2.0(1.5-4.4)$ \\
\hline C-reactive protein, $\mathrm{mg} / \mathrm{L}$ & $117.7(68.4-189.0)$ \\
\hline Procalcitonin, $\mathrm{ng} / \mathrm{mL}$ & $1.4(0.3-4.3)$ \\
\hline Pneumothorax & $22(18.5)$ \\
\hline Tracheostomy & $45(37.8)$ \\
\hline 28-day mortality & $45(37.8)$ \\
\hline \multicolumn{2}{|c|}{ Data are presented by numbers (\%) or median (IQR) unless indicated otherwise. } \\
\hline \multicolumn{2}{|c|}{$\begin{array}{l}\text { IQR, interquartile range; BMI, body mass index; ILD, interstitial lung disease; CRRT, continuous renal } \\
\text { replacement therapy; AKI, acute kidney injury; APACHE II, Acute Physiology and Chronic Health } \\
\text { Evaluation II; SOFA, Sequential Organ Failure Assessment; SAPS II, simplified acute physiology score } \\
\text { II; WBC, white blood cell; BUN, blood urea nitrogen }\end{array}$} \\
\hline
\end{tabular}

\section{Factors and outcomes associated with occurrence of pneumothorax}

In total, 22 patients (18.5\%) developed pneumothorax during MV treatment, with the median time from initiating ventilator management to development of pneumothorax being 8.0 days (IQR, 1.5-16.0). Table 2shows comparisons of associated factors and outcomes, according to occurrence of pneumothorax. On univariate analysis, low body mass index (BMI), underlying airway disease, renal failure requiring CRRT, low procalcitonin level, low SOFA score, and CMV antigenemia were associated with the development of pneumothorax in patients with PCP and respiratory failure. However, there was no significant difference in MV parameters within 24 after MV initiation, including tidal volume, peak pressure, and dynamic driving pressure. Peak pressure at three days after MV initiation and maximal peak pressure seven days after MV initiation did not show statistical significance between those with and without pneumothorax. Furthermore, there was no significant difference in proportion of patients who had undergone tracheostomy and mortality rate, according to presence or absence of pneumothorax. 
Table 2

Comparison of characteristics according to occurrence of pneumothorax

\begin{tabular}{|c|c|c|c|c|c|}
\hline & \multirow{2}{*}{$\begin{array}{l}\begin{array}{l}\text { No } \\
\text { pneumothorax }\end{array} \\
\begin{array}{l}\mathrm{N}=97(81.5 \\
\%)\end{array}\end{array}$} & \multirow{2}{*}{$\begin{array}{l}\text { Pneumothorax } \\
\mathrm{N}=22 \text { (18.5\%) }\end{array}$} & \multirow{2}{*}{$\begin{array}{l}p- \\
\text { value }\end{array}$} & \multicolumn{2}{|c|}{ Multivariate analysis } \\
\hline & & & & OR $(95 \% \mathrm{Cl})$ & $\begin{array}{l}p- \\
\text { value }\end{array}$ \\
\hline Median age, years & $\begin{array}{l}65.0(55.0- \\
71.5)\end{array}$ & $\begin{array}{l}64.5(58.0- \\
72.0)\end{array}$ & 0.760 & $\begin{array}{l}1.0(0.958- \\
1.047)\end{array}$ & 0.949 \\
\hline Sex (male) & $62(63.9)$ & $14(63.6)$ & 0.980 & $\begin{array}{l}1.3(0.440- \\
3.895)\end{array}$ & 0.628 \\
\hline Height, cm & $\begin{array}{l}163.0(156.0- \\
169.5)\end{array}$ & $\begin{array}{l}165.0(156.8- \\
170.8)\end{array}$ & 0.415 & & \\
\hline $\mathrm{BMI}, \mathrm{kg} / \mathrm{m}^{2}$ & $\begin{array}{l}22.7(20.5- \\
25.7)\end{array}$ & $\begin{array}{l}20.8(19.5- \\
23.0)\end{array}$ & 0.012 & $\begin{array}{l}1.2(1.011- \\
1.356)\end{array}$ & 0.035 \\
\hline Ever smoker & $28(28.9)$ & $6(27.3)$ & 0.881 & & \\
\hline Charlson Comorbidity Index & $3(2-4)$ & $2(2-3.5)$ & 0.447 & & \\
\hline \multicolumn{6}{|l|}{ Underlying disease } \\
\hline Congestive heart failure & $8(8.2)$ & $3(13.6)$ & 0.431 & & \\
\hline Chronic lung disease - airway & $5(5.2)$ & $4(18.2)$ & 0.037 & $\begin{array}{l}0.2(0.050- \\
1.160)\end{array}$ & 0.076 \\
\hline Chronic lung disease - ILD & $17(17.5)$ & $5(22.7)$ & 0.553 & & \\
\hline Chronic kidney disease & $27(27.8)$ & $4(18.2)$ & 0.352 & & \\
\hline Chronic liver disease & $8(8.2)$ & $1(4.5)$ & 1.0 & & \\
\hline Solid cancer & $31(32.0)$ & $6(27.3)$ & 0.801 & & \\
\hline Hematologic malignancy & $20(20.6)$ & $2(9.1)$ & 0.360 & & \\
\hline History of lung operation & $8(8.2)$ & $5(22.7)$ & 0.063 & & \\
\hline History of pneumothorax & $0(0)$ & $1(4.5)$ & 0.185 & & \\
\hline CRRT due to AKI & $38(39.2)$ & $3(13.6)$ & 0.023 & & \\
\hline
\end{tabular}

Data were presented by numbers (\%) or median (IQR) unless otherwise indicated.

IQR, interquartile range; $B M I$, body mass index; ILD, interstitial lung disease; CRRT, continuous renal replacement therapy; $\mathrm{AKI}$, acute kidney injury; $\mathrm{PaO}$ 2, partial pressure of arterial oxygen; $\mathrm{FiO} 2$, fraction of inspired 02 concentration; APACHE II, Acute Physiology and Chronic Health Evaluation II; SOFA, Sequential Organ Failure Assessment; SAPS II, simplified acute physiology score II; WBC, white blood cell; BUN, blood urea nitrogen 


\begin{tabular}{|c|c|c|c|c|c|}
\hline & \multirow{2}{*}{$\begin{array}{l}\begin{array}{l}\text { No } \\
\text { pneumothorax }\end{array} \\
\begin{array}{l}\mathrm{N}=97(81.5 \\
\%)\end{array}\end{array}$} & \multirow{2}{*}{$\begin{array}{l}\text { Pneumothorax } \\
\mathrm{N}=22 \text { (18.5\%) }\end{array}$} & \multirow{2}{*}{$\begin{array}{l}p- \\
\text { value }\end{array}$} & \multicolumn{2}{|c|}{ Multivariate analysis } \\
\hline & & & & OR $(95 \% \mathrm{Cl})$ & $\begin{array}{l}p- \\
\text { value }\end{array}$ \\
\hline WBC, $\times 10^{3} / \mu \mathrm{L}$ & $9.9(5.7-14.8)$ & $\begin{array}{l}11.5(6.1- \\
22.4)\end{array}$ & 0.162 & & \\
\hline Lactate, mmol/L & $2.2(1.5-5.1)$ & $1.8(1.4-2.1)$ & 0.130 & & \\
\hline C-reactive protein, mg/L & $\begin{array}{l}129.2(69.9- \\
208.2)\end{array}$ & $\begin{array}{l}100.5(10.1- \\
160.2)\end{array}$ & 0.108 & & \\
\hline Procalcitonin, ng/mL & $1.5(0.5-5.2)$ & $0.3(0.2-0.5)$ & 0.008 & & \\
\hline $\mathrm{PaO}_{2} / \mathrm{FiO}_{2}$ ratio & $\begin{array}{l}83.6(66.7- \\
111.0)\end{array}$ & $\begin{array}{l}79.4(61.6- \\
101.1)\end{array}$ & 0.372 & & \\
\hline APACHE II score & $27(21-32)$ & $21(18-32)$ & 0.129 & & \\
\hline SOFA score & $8.0(6.0-12.0)$ & $7.0(4.5-8.0)$ & 0.003 & & \\
\hline SAPSII & $\begin{array}{l}37.0(30.0- \\
51.0)\end{array}$ & $\begin{array}{l}35.0(24.5- \\
45.0)\end{array}$ & 0.134 & & \\
\hline Cytomegalovirus antigenemia & $54(55.7)$ & $21(95.5)$ & $<0.001$ & $\begin{array}{l}13.9 \\
(1.737- \\
111.072)\end{array}$ & 0.013 \\
\hline Tracheostomy & $34(35.1)$ & $11(50)$ & 0.192 & & \\
\hline 28-day mortality & $38(39.2)$ & $7(31.8)$ & 0.521 & & \\
\hline In-hospital mortality & $68(70.1)$ & 17 (77.3) & 0.502 & & \\
\hline \multicolumn{6}{|l|}{$\begin{array}{l}\text { Mechanical ventilator } \\
\text { parameters }\end{array}$} \\
\hline Respiratory rate, / min & $\begin{array}{l}20.0(16.0- \\
24.0)\end{array}$ & $\begin{array}{l}20.0(18.0- \\
26.0)\end{array}$ & 0.379 & & \\
\hline $\begin{array}{l}\text { Tidal volume at admission day, } \\
\mathrm{mL} / \mathrm{kg}\end{array}$ & $6.7(6.1-7.6)$ & $6.8(6.2-7.5)$ & 0.405 & & \\
\hline $\begin{array}{l}\text { Peak pressure at admission } \\
\text { day, } \mathrm{cmH}_{2} \mathrm{O}\end{array}$ & $\begin{array}{l}25.0(22.0- \\
30.0)\end{array}$ & $\begin{array}{l}26.0(22.8- \\
32.3)\end{array}$ & 0.420 & & \\
\hline
\end{tabular}

Data were presented by numbers (\%) or median (IQR) unless otherwise indicated.

IQR, interquartile range; $B M I$, body mass index; ILD, interstitial lung disease; CRRT, continuous renal replacement therapy; $\mathrm{AKI}$, acute kidney injury; $\mathrm{PaO} 2$, partial pressure of arterial oxygen; FiO2, fraction of inspired 02 concentration; APACHE II, Acute Physiology and Chronic Health Evaluation II; SOFA, Sequential Organ Failure Assessment; SAPS II, simplified acute physiology score II; WBC, white blood cell; BUN, blood urea nitrogen 


\begin{tabular}{|c|c|c|c|c|c|}
\hline & \multirow{2}{*}{$\begin{array}{l}\begin{array}{l}\text { No } \\
\text { pneumothorax }\end{array} \\
\begin{array}{l}\mathrm{N}=97(81.5 \\
\text { \%) }\end{array}\end{array}$} & \multirow{2}{*}{$\begin{array}{l}\text { Pneumothorax } \\
\mathrm{N}=22(18.5 \%)\end{array}$} & \multirow{2}{*}{$\begin{array}{l}p- \\
\text { value }\end{array}$} & \multicolumn{2}{|c|}{ Multivariate analysis } \\
\hline & & & & OR $(95 \% \mathrm{Cl})$ & $\begin{array}{l}p- \\
\text { value }\end{array}$ \\
\hline $\begin{array}{l}\text { Peak pressure at 3-day after } \\
\text { admission, } \mathrm{cmH}_{2} \mathrm{O}\end{array}$ & $\begin{array}{l}26.0(20.3- \\
30.0)\end{array}$ & $\begin{array}{l}26.0(21.0- \\
34.5)\end{array}$ & 0.357 & & \\
\hline $\begin{array}{l}\text { Maximal peak pressure, } \\
\mathrm{cmH}_{2} \mathrm{O}\end{array}$ & $\begin{array}{l}29.0(25.0- \\
34.0)\end{array}$ & $\begin{array}{l}31.5(26.0- \\
38.5)\end{array}$ & 0.205 & & \\
\hline $\begin{array}{l}\text { Positive expiratory end } \\
\text { pressure, } \mathrm{cmH}_{2} \mathrm{O}\end{array}$ & $7.0(5.0-10.0)$ & $5.5(5.0-8.0)$ & 0.069 & & \\
\hline $\begin{array}{l}\text { Dynamic driving pressure, } \\
\mathrm{CmH}_{2} \mathrm{O}\end{array}$ & $\begin{array}{l}19.0(15.0- \\
26.8)\end{array}$ & $\begin{array}{l}17.0(14.0- \\
22.0)\end{array}$ & 0.166 & & \\
\hline \multicolumn{6}{|c|}{ Data were presented by numbers (\%) or median (IQR) unless otherwise indicated. } \\
\hline \multicolumn{6}{|c|}{$\begin{array}{l}\text { IQR, interquartile range; BMI, body mass index; ILD, interstitial lung disease; CRRT, continuous renal } \\
\text { replacement therapy; AKI, acute kidney injury; PaO2, partial pressure of arterial oxygen; FiO2, fraction } \\
\text { of inspired O2 concentration; APACHE II, Acute Physiology and Chronic Health Evaluation II; SOFA, } \\
\text { Sequential Organ Failure Assessment; SAPS II, simplified acute physiology score II; WBC, white blood } \\
\text { cell; BUN, blood urea nitrogen }\end{array}$} \\
\hline
\end{tabular}

Age, sex, and variables related to pulmonary involvement, including BMI, underlying airway disease, and CMV antigenemia, with a $p$-value $<0.05$ in the univariate analysis were used in the multivariate analysis. Low BMI (OR, 1.2; 95\%67 Cl, 1.011-1.356) and CMV antigenemia (OR 13.9; 95\% Cl, 1.737-111.072) remained significant risk factors in the multivariate analysis.

\section{Prognosis of PCP and associated risks}

The 28-day mortality rate was $62.2 \%(n=45)$. The in-hospital mortality rate was $71.4 \%(n=85)$. Patients with renal failure requiring CRRT were at significantly higher mortality $(p=0.001)$. High disease severity, including high APACHE II score $(p=0.045)$, SOFA score $(p<0.001)$, and SAPS II $(p=0.015)$, was related to poor outcomes in patients with PCP. Regarding MV parameters, the peak pressure three days after admission $(p=0.003)$ and maximal peak pressure $(p=0.009)$ were higher in non-survivors than in survivors. In the multivariate analysis, renal failure requiring CRRT (OR, 6.4; $95 \% \mathrm{Cl}, 1.905-21.835)$ and peak pressure at three days after admission $(\mathrm{OR}, 0.849 ; 95 \% \mathrm{Cl}, 0.732-0.985)$ were significantly associated with poor prognosis in patients with PCP and respiratory failure. (Table 3 ) 
Table 3

Comparison of the characteristics between survivors and non-survivors

\begin{tabular}{|c|c|c|c|c|c|}
\hline & \multirow{2}{*}{$\begin{array}{l}\text { Survivor } \\
\begin{array}{l}N=74(62.2 \\
\%)\end{array}\end{array}$} & \multirow{2}{*}{$\begin{array}{l}\text { Non-survivor } \\
\begin{array}{l}N=45 \\
(37.8 \%)\end{array}\end{array}$} & \multirow{2}{*}{$\begin{array}{l}p- \\
\text { value }\end{array}$} & \multicolumn{2}{|c|}{ Multivariate analysis } \\
\hline & & & & OR $(95 \% \mathrm{Cl})$ & $\begin{array}{l}p- \\
\text { value }\end{array}$ \\
\hline Median age & $60.9(57-72)$ & $\begin{array}{l}64(55.5- \\
71.5)\end{array}$ & 0.587 & & \\
\hline Sex (male) & $48(64.9)$ & $28(62.2)$ & 0.771 & $\begin{array}{l}1.158(0.423- \\
3.169)\end{array}$ & 0.775 \\
\hline Height, cm & $\begin{array}{l}164.0 \\
(156.0- \\
170.0)\end{array}$ & $\begin{array}{l}161.8 \\
(156.0-168)\end{array}$ & 0.616 & & \\
\hline $\mathrm{BMI}, \mathrm{kg} / \mathrm{m}^{2}$ & $\begin{array}{l}22.4(20.4- \\
25.4)\end{array}$ & $\begin{array}{l}22.1(19.8- \\
24.1)\end{array}$ & 0.511 & & \\
\hline Ever smoker & $21(28.4)$ & $13(28.9)$ & 0.952 & & \\
\hline Charlson Comorbidity Index & $3(2-4)$ & $3(2-5.5)$ & 0.281 & & \\
\hline \multicolumn{6}{|l|}{ Comorbidity disease } \\
\hline Congestive heart failure & $4(5.4)$ & $7(15.6)$ & 0.100 & & \\
\hline Chronic lung disease - airway & $4(5.4)$ & $5(11.1)$ & 0.296 & & \\
\hline Chronic lung disease - ILD & $15(20.3)$ & $7(15.6)$ & 0.521 & & \\
\hline Chronic kidney disease & $17(23)$ & $14(31.1)$ & 0.327 & & \\
\hline Chronic liver disease & $6(8.1)$ & $3(6.7)$ & 1.0 & & \\
\hline Solid cancer & $21(28.4)$ & $16(35.6)$ & 0.412 & & \\
\hline Hematologic malignancy & $12(16.2)$ & $10(22.2)$ & 0.413 & & \\
\hline CRRT due to AKI & $17(23)$ & $24(53.3)$ & 0.001 & $\begin{array}{l}6.447(1.905- \\
21.835)\end{array}$ & 0.003 \\
\hline \multicolumn{6}{|l|}{ Clinical parameters } \\
\hline WBC, $x 10^{3} / \mu \mathrm{L}$ & $\begin{array}{l}10.8(6.8- \\
15.7)\end{array}$ & $\begin{array}{l}8.5(4.0- \\
14.6)\end{array}$ & 0.070 & & \\
\hline $\mathrm{Hct}, \%$ & $\begin{array}{l}28.0(24.9- \\
32.6)\end{array}$ & $\begin{array}{l}25.6(22.9- \\
29.6)\end{array}$ & 0.045 & & \\
\hline \multicolumn{6}{|c|}{ Data are presented as numbers (\%) or median (IQR) unless indicated otherwise. } \\
\hline \multicolumn{6}{|c|}{$\begin{array}{l}\text { IQR, interquartile range; BMI, body mass index; ILD, interstitial lung disease; CRRT, continuous renal } \\
\text { replacement therapy; AKI, acute kidney injury; APACHE II, Acute Physiology and Chronic Health } \\
\text { Evaluation II; SOFA, Sequential Organ Failure Assessment; SAPS II, simplified acute physiology score } \\
\text { II; WBC, white blood cell; BUN, blood urea nitrogen }\end{array}$} \\
\hline
\end{tabular}




\begin{tabular}{|c|c|c|c|c|c|}
\hline & \multirow{2}{*}{$\begin{array}{l}\text { Survivor } \\
\begin{array}{l}N=74(62.2 \\
\%)\end{array}\end{array}$} & \multirow{2}{*}{$\begin{array}{l}\text { Non-survivor } \\
\begin{array}{l}N=45 \\
(37.8 \%)\end{array}\end{array}$} & \multirow{2}{*}{$\begin{array}{l}p- \\
\text { value }\end{array}$} & \multicolumn{2}{|c|}{ Multivariate analysis } \\
\hline & & & & OR $(95 \% \mathrm{Cl})$ & $\begin{array}{l}p- \\
\text { value }\end{array}$ \\
\hline Platelet, $\times 10^{3} / \mu \mathrm{L}$ & $\begin{array}{l}153.5(79.5- \\
263)\end{array}$ & $79(28-179)$ & 0.001 & $\begin{array}{l}1.000 \\
(0.996- \\
1.004)\end{array}$ & 0.911 \\
\hline $\mathrm{BUN}, \mathrm{mg} / \mathrm{dL}$ & $\begin{array}{l}27.2(18.6- \\
39.4)\end{array}$ & $\begin{array}{l}35.2(22.9- \\
52.0)\end{array}$ & 0.069 & & \\
\hline Creatinine, $\mathrm{mg} / \mathrm{dL}$ & $1.1(0.6-1.8)$ & $1.1(0.6-2)$ & 0.810 & & \\
\hline Albumin, $\mathrm{g} / \mathrm{dL}$ & $2.5(2.3-2.7)$ & $2.3(2.0-2.6)$ & 0.082 & & \\
\hline Total bilirubin, $\mathrm{mg} / \mathrm{dL}$ & $0.5(0.3-1.0)$ & $0.9(0.4-3.4)$ & 0.019 & $\begin{array}{l}0.744 \\
(0.443- \\
1.249)\end{array}$ & 0.263 \\
\hline Sodium, $\mathrm{mmol} / \mathrm{L}$ & $\begin{array}{l}135(132- \\
138)\end{array}$ & $\begin{array}{l}139.5 \\
(132.8- \\
141.8)\end{array}$ & 0.248 & & \\
\hline Potassium, mmol/L & $4.4(3.6-5.0)$ & $4.4(3.8-5.0)$ & 0.855 & & \\
\hline Lactate, $\mathrm{mmol} / \mathrm{L}$ & $1.8(1.2-4.3)$ & $\begin{array}{l}3.1(1.5- \\
13.1)\end{array}$ & 0.054 & & \\
\hline Procalcitonin, ng/mL & $1.4(0.3-5.1)$ & $\begin{array}{l}1.4(0.3- \\
10.9)\end{array}$ & 0.556 & & \\
\hline C-reactive protein, $\mathrm{mg} / \mathrm{L}$ & $\begin{array}{l}113.6(50.4- \\
182.1)\end{array}$ & $\begin{array}{l}137.9(71.7- \\
227.8)\end{array}$ & 0.253 & & \\
\hline APACHE II score & $\begin{array}{l}25(19.0- \\
30.0)\end{array}$ & $\begin{array}{l}28.5(22.5- \\
37.0)\end{array}$ & 0.045 & $\begin{array}{l}0.98(0.913- \\
1.047)\end{array}$ & 0.519 \\
\hline SOFA score & $7(6-9)$ & $10(8-13)$ & $\begin{array}{l}< \\
0.001\end{array}$ & $\begin{array}{l}1.03(0.873- \\
1.342)\end{array}$ & 0.469 \\
\hline SAPS II & $\begin{array}{l}35.0(27- \\
45.5)\end{array}$ & $44(33-54)$ & 0.015 & & \\
\hline Pneumothorax & $15(20.3 \%)$ & $7(15.6 \%)$ & 0.521 & & \\
\hline Cytomegalovirus antigenemia & $51(68.9)$ & $24(53.3)$ & 0.088 & & \\
\hline $\begin{array}{l}\text { Mechanical ventilator } \\
\text { parameters }\end{array}$ & & & & & \\
\hline
\end{tabular}

Data are presented as numbers (\%) or median (IQR) unless indicated otherwise.

IQR, interquartile range; BMI, body mass index; ILD, interstitial lung disease; CRRT, continuous renal replacement therapy; AKI, acute kidney injury; APACHE II, Acute Physiology and Chronic Health Evaluation II; SOFA, Sequential Organ Failure Assessment; SAPS II, simplified acute physiology score II; WBC, white blood cell; BUN, blood urea nitrogen 


\begin{tabular}{|c|c|c|c|c|c|}
\hline & \multirow{2}{*}{$\begin{array}{l}\text { Survivor } \\
\begin{array}{l}\mathrm{N}=74(62.2 \\
\%)\end{array}\end{array}$} & \multirow{2}{*}{$\begin{array}{l}\text { Non-survivor } \\
\begin{array}{l}N=45 \\
(37.8 \%)\end{array}\end{array}$} & \multirow{2}{*}{$\begin{array}{l}p- \\
\text { value }\end{array}$} & \multicolumn{2}{|c|}{ Multivariate analysis } \\
\hline & & & & OR $(95 \% \mathrm{Cl})$ & $\begin{array}{l}p- \\
\text { value }\end{array}$ \\
\hline Respiratory rate, /min & $\begin{array}{l}20(16.0- \\
25.3)\end{array}$ & $\begin{array}{l}20(18.0- \\
24.0)\end{array}$ & 0.535 & & \\
\hline $\begin{array}{l}\text { Tidal volume at admission day, } \\
\mathrm{mL} / \mathrm{kg}\end{array}$ & $\begin{array}{l}380.0 \\
(320.0- \\
400.0)\end{array}$ & $\begin{array}{l}350(300.0- \\
380.0)\end{array}$ & 0.831 & & \\
\hline $\begin{array}{l}\text { Peak pressure at admission } \\
\text { day, } \mathrm{CmH}_{2} \mathrm{O}\end{array}$ & $\begin{array}{l}27.0(20.8- \\
32.0)\end{array}$ & $\begin{array}{l}26.0(23.0- \\
33.0)\end{array}$ & 0.988 & & \\
\hline $\begin{array}{l}\text { Peak pressure at 3-day after } \\
\text { admission, } \mathrm{CmH}_{2} \mathrm{O}\end{array}$ & $\begin{array}{l}25.0(19.0- \\
31.3)\end{array}$ & $\begin{array}{l}31.0(27.0- \\
41.0)\end{array}$ & 0.003 & $\begin{array}{l}0.85(0.732- \\
0.985)\end{array}$ & 0.030 \\
\hline Maximal peak pressure, $\mathrm{cmH}_{2} \mathrm{O}$ & $\begin{array}{l}30.0(24.5- \\
35.5)\end{array}$ & $\begin{array}{l}36.0(29.0- \\
42.0)\end{array}$ & 0.009 & $\begin{array}{l}0.98(0.846- \\
1.128)\end{array}$ & 0.977 \\
\hline $\begin{array}{l}\text { Positive expiratory end } \\
\text { pressure, } \mathrm{cmH}_{2} \mathrm{O}\end{array}$ & $5.0(5.0-8.0)$ & $\begin{array}{l}7.0(5.0- \\
10.0)\end{array}$ & 0.328 & & \\
\hline $\begin{array}{l}\text { Dynamic driving pressure, } \\
\mathrm{cmH}_{2} \mathrm{O}\end{array}$ & $\begin{array}{l}18.0(14.8- \\
22.3)\end{array}$ & $\begin{array}{l}17.0(14.0- \\
22.0)\end{array}$ & 0.777 & & \\
\hline \multicolumn{6}{|c|}{ Data are presented as numbers (\%) or median (IQR) unless indicated otherwise. } \\
\hline \multicolumn{6}{|c|}{$\begin{array}{l}\text { IQR, interquartile range; BMI, body mass index; ILD, interstitial lung disease; CRRT, continuous renal } \\
\text { replacement therapy; AKI, acute kidney injury; APACHE II, Acute Physiology and Chronic Health } \\
\text { Evaluation II; SOFA, Sequential Organ Failure Assessment; SAPS II, simplified acute physiology score } \\
\text { Il; WBC, white blood cell; BUN, blood urea nitrogen }\end{array}$} \\
\hline
\end{tabular}

\section{Final prognosis of surviving patients}

Of 119 patients, 45 (37.8\%) underwent tracheostomy due to weaning failure. The need for tracheostomy for long term MV management did not significantly differ between patients with- and without pneumothorax (Table 2).

We further investigated on the 74 study patients who survived more than 28 days. Among them, 15 (20.3\%) developed pneumothorax. There was no significant difference in tracheostomy rates between those with and without pneumothorax (Table 4). However, patients without pneumothorax were significantly more successful in weaning from MV than were patients with pneumothorax (44\% vs. $13.3 \%$, $p=0.037$ ). The ICU length of stay was also longer in patients with pneumothorax, although there was no statistically significant difference $(p=0.068)$. Patients with PCP and pneumothorax were significantly associated with a poor prognosis, with a mortality of $33.3 \%(p=0.048)$, and only two patients $(13.3 \%)$ 
were eventually discharged home, compared to 25 (51.0\%) successful home discharges among patients without pneumothorax $(p=0.010$, Table 4$)$.

Table 4

Clinical prognosis in patients with 28-day survival $(n=74)$

\begin{tabular}{|llll|}
\hline & No pneumothorax & Pneumothorax & \\
\hline Tracheostomy & $\mathrm{N}=59(79.7)$ & $\mathrm{N}=15(20.3)$ & $p$-value \\
\hline Ventilator weaning & $29(49.2)$ & $9(60)$ & 0.453 \\
\hline ICU length of stay & $26(44)$ & $2(13.3)$ & 0.037 \\
\hline Survivors & $18(12-40)$ & $32(24-58)$ & 0.068 \\
\hline Discharge to home & $29(49.2)$ & $5(33.3)$ & 0.048 \\
\hline $\begin{array}{l}\text { Data are presented as numbers }(\%) \text { or median (IQR) unless indicated otherwise. ICU, intensive care } \\
\text { unit }\end{array}$ & $25(51.0)$ & 0.010 \\
\hline
\end{tabular}

\section{Discussion}

This study described the incidence and related factors of pneumothorax in patients with PCP and their prognosis. In addition, we analyzed survival outcomes in patients with PCP and acute respiratory failure. The development of pneumothorax in PCP was not associated with increased 28-day mortality; however, patients with pneumothorax had difficulty and prolonged MV weaning. Among several factors, CMV coinfection was associated with the development of pneumothorax.

There are several complications associated with PCP [11]. Among them, pneumothorax occurs with prevalence of $5-20 \%$ [12], and is known to occur frequently in patients with conditions leading to increased intrathoracic pressure, including airway disease and interstitial lung disease [7]. Pneumothorax is also a common complication during ventilator treatment, with a reported incidence of $4-15 \%$ [13-15]. Patients with acute respiratory distress syndrome are more vulnerable to the occurrence of pneumothorax, with further higher risks in patients with underlying lung disease, than those who do not have this syndrome [16]. In our study population of patients with acute respiratory failure requiring MV care, which is a risk factor for pneumothorax [17], the incidence of pneumothorax was $18.5 \%(22 / 119)$, which is high. Furthermore, underlying lung diseases, including airway disease and interstitial lung disease, showed relation to increased occurrence of pneumothorax in the univariate analysis, in accordance with a previous report [18].

We found that there were no significant differences in MV parameters between the patients with- and without pneumothorax. Some studies showed that ventilator parameters, such as peak airway pressure, tidal volume, and PEEP, had no correlation with increased risk of pneumothorax [19-21], although earlier 
studies had reported relevance [22]. Miller et al reported that protective lung strategies had an effect on decreasing barotrauma [23]. We had managed patients with tidal volumes of 6-7 mg/ $\mathrm{kg}$ and peak pressures lower than $35 \mathrm{cmH}_{2} \mathrm{O}$, according to the lung protective strategy [24]. We showed that respiratory mechanics did not significantly affect the development of pneumothorax in patients with PCP when this lung protective strategy was applied. Furthermore, in one study, Boussarsar et al. said that barotrauma during $\mathrm{MV}$ care was more strongly associated with underlying lung conditions and compliance than with MV parameters [25].

In immunocompromised patients, PCP commonly presents with co-infection of other pathogens, especially CMV [26]. The reports on effect and outcome of CMV co-infection with PCP are controversial $[27,28]$, although there are several researches stating that concurrent infection of $C M V$ is related to increased mortality and poor prognosis $[29,30]$. Our study showed that CMV antigenemia was not associated with a high mortality rate; however, patients with CMV antigenemia were significantly associated with increased occurrence of pneumothorax. In our study populations, the effect of CMV reactivation on PCP prognosis is meaningful because the seropositivity of CMV in Koreans was reported as high as $94.1 \%$ [31]. Cook et al. suggested that CMV reactivation could cause abnormal cytokine/chemokine expression, resulting in pulmonary fibrosis in an animal model [32]. In one prior research on histopathological findings in 12 deceased patients with PCP, three had evidence of CMV coinfection, and two of them presenting with pulmonary fibrosis [33]. Furthermore, structural changes in lung parenchyma, including fibrosis, is one of proposed mechanisms of pneumothorax and increases vulnerability to the occurrence of pneumothorax [34].

Occurrence of PCP in patients without HIV has poorer progress and higher mortality than that in patients with HIV [35]. In our study, the in-hospital mortality rate was high at $71.4 \%$, will only 28 patients $(23.5 \%)$ achieving MV weaning. Pneumothorax in patients with PCP with- or without HIV was difficult to treat and had a worse prognosis than pneumothorax from other etiologies $[9,17,36]$. However, in our study, the development of pneumothorax was not associated with increased mortality. In a previous study, acute respiratory failure requiring invasive $\mathrm{MV}$ was found to be a risk factor for increased mortality; therefore, the effect of pneumothorax was likely to be lessened because all patients enrolled our study already had respiratory failure [35]. However, we found that development of pneumothorax in patients with PCP who required invasive $\mathrm{MV}$ procedures made weaning difficult.

Our study had several limitations. First, this study was retrospectively conducted at a single center. However, we enrolled a large number of patients with PCP and respiratory failure without HIV requiring invasive MV procedures. Furthermore, since all enrolled patients were applied MV, the bias of applying MV could be reduced. Second, we did not acquire microbiological findings from patient samples for diagnosis of PCP but only diagnosed via P. jirovecii PCR assays. However, the sensitivity and specificity of this assay for detecting $P$. jirovecii were comparable to those of microscopic staining [37]. Furthermore, we included only patients who were treated for PCP with typical symptoms of pneumonia and characteristic radiological findings. Third, in patients discharged to long-term outpatient care after survival, it was not possible to investigate final success of MV weaning.

Page $15 / 20$ 
Nevertheless, our study also had some strengths. We analyzed a large number of patients with PCP and acute respiratory failure, and found related factor of pneumothorax. ICU clinicians might predict that patients with PCP and CMV antigenemia have increased risk of pneumothorax. Additionally, development of pneumothorax in patients with PCP could be a predictive factor of delayed MV weaning and poor final outcome. These results could be helpful to the real clinical field ICU clinician. However, further prospective studies are needed to validate our findings.

The results of this study suggest that pneumothorax development in patients with PCP and acute respiratory failure was not associated with increased 28-day mortality. However, patients with PCP who develop pneumothorax might have difficulty and delayed weaning from MV. Concomitant CMV antigenemia could be a predictive factor for pneumothorax occurrence. Therefore, clinicians need to closely observe the occurrence of pneumothorax in patient with PCP and CMV antigenemia and should anticipate that MV weaning may be difficult in such patients.

\section{Abbreviations}

PCP, Pneumocystis jirovecii pneumonia; ICU, intensive care unit; HIV, human immunodeficiency virus; APACHE, acutely physiology and chronic health evaluation; PCR, polymerase chain reaction; $C T$, computed tomography; SOFA, Sequential Organ Failure Assessment; SAPS, Simplified Acute Physiology Score; MV, mechanical ventilator; PEEP, positive expiratory end pressure; IQRs, interquartile ranges; ORs, Odds ratios; Cls, confidence intervals; CRRT, continuous renal replacement therapy; CMV, cytomegalovirus; BMI, body mass index

\section{Declarations}

Ethics approval: Approval for this study was provided by the institutional review board of Yongin Severance Hospital (IRB 9-2021-0045). The need for informed consent was waived due to the retrospective nature of this study. This study was conducted in accordance with the tenets set by the Declaration of Helsinki.

Consent for publication: Not applicable.

Availability of data and materials: The datasets generated during and/or analysed during the current study are available from the corresponding author on reasonable request.

Competing interests: The authors declare that they have no competing interests.

Funding: This work was supported by the Korea Medical Device Development Fund grant funded by the Korean government (the Ministry of Science and ICT, the Ministry of Trade, Industry and Energy, the Ministry of Health \& Welfare, the Ministry of Food and Drug Safety) (Project Number: 202011B26). 
Author's contributions: Conceptualization: J.S.C., S.H.L., Data curation: J.S.C., S.H.L., S.H.Y., A.Y.L., S.Y.K., S.H.L., K.S.C., E.Y.K., J.Y.J., Y.A.K., M.S.P., Y.S.K., Formal analysis: J.S.C., M.C.K., C.H.S., S.H.L, Funding acquisition: A.Y.L., K.S.C., Y.S.K., S.H.L., Investigation: J.S.C., S.H.K., M.C.K., C.H.S., S.R.K., B.H.P., E.H.L., S.H.L, Methodology: J.S.C., S.H.L, Supervision: E.H.L., M.S.P., Y.S.K., S.H.L., Writing - original draft: J.S.C., S.H.L, Writing - review \& editing: All authors reviewed and edited the manuscript

ACKNOWLEDGEMENTS: Not applicable.

\section{References}

1. Varthalitis I, Aoun M, Daneau D, Meunier F: Pneumocystis carinii pneumonia in patients with cancer. An increasing incidence. Cancer 1993, 71(2):481-485.

2. Kim SJ, Lee J, Cho YJ, Park YS, Lee CH, Yoon HI, Lee SM, Yim JJ, Lee JH, Yoo CG et al: Prognostic factors of Pneumocystis jirovecii pneumonia in patients without HIV infection. J Infect 2014, 69(1):88-95.

3. Boonsarngsuk V, Sirilak S, Kiatboonsri S: Acute respiratory failure due to Pneumocystis pneumonia: outcome and prognostic factors. Int J Infect Dis 2009, 13(1):59-66.

4. Tumbarello M, Tacconelli E, Pirronti T, Cauda R, Ortona L: Pneumothorax in HIV-infected patients: role of Pneumocystis carinii pneumonia and pulmonary tuberculosis. Eur Respir J 1997, 10(6):13321335.

5. Kotani T, Katayama S, Miyazaki Y, Fukuda S, Sato Y, Ohsugi K: Risk Factors for the Mortality of $<i>$ Pneumocystis jirovecii</i > Pneumonia in Non-HIV Patients Who Required Mechanical Ventilation: A Retrospective Case Series Study. BioMed Research Internationa/ 2017, 2017:7452604.

6. McClellan MD, Miller SB, Parsons PE, Cohn DL: Pneumothorax with Pneumocystis carinii Pneumonia in AIDS: Incidence and Clinical Characteristics. Chest 1991, 100(5):1224-1228.

7. Bedos J-P, Dumoulin J-L, Gachot B, Veber B, Wolff M, Regnier B, Chevret S: Pneumocystis carinii pneumonia requiring intensive care management: Survival and prognostic study in 110 patients with human immunodeficiency virus. Critical Care Medicine 1999, 27(6):1109-1115.

8. Coker RJ, Moss F, Peters B, McCarty M, Nieman R, Claydon E, Mitchell D, Harris JR: Pneumothorax in patients with AIDS. Respir Med 1993, 87(1):43-47.

9. Festic E, Gajic O, Limper AH, Aksamit TR: Acute Respiratory Failure Due to Pneumocystis Pneumonia in Patients Without Human Immunodeficiency Virus Infection. Chest 2005, 128(2):573-579.

10. Weinberg A, Hodges TN, Li S, Cai G, Zamora MR: Comparison of PCR, antigenemia assay, and rapid blood culture for detection and prevention of cytomegalovirus disease after lung transplantation. $J$ Clin Microbio/ 2000, 38(2):768-772.

11. Teruya K, Yasuoka A, Yamaguchi M, Yasuoka C, Yamamoto Y, Genka I, Tachikawa N, Kikuchi Y, Oka $\mathrm{S}$ : Complications during clinical courses of Pneumocystis carinii pneumonia in patients with acquired immunodeficiency syndrome. Intern Med 2001, 40(3):221-226. 
12. Ko Y, Jeong B-H, Park HY, Koh W-J, Suh GY, Chung MP, Kwon OJ, Jeon K: Outcomes of Pneumocystis pneumonia with respiratory failure in HIV-negative patients. Journal of Critical Care 2014, 29(3):356361.

13. Petersen GW, Baier H: Incidence of pulmonary barotrauma in a medical ICU. Critical Care Medicine 1983, 11(2).

14. Zwillich CW, Pierson DJ, Creagh CE, Sutton FD, Schatz E, Petty TL: Complications of assisted ventilation: A prospective study of 354 consecutive episodes. The American Journal of Medicine 1974, 57(2):161-170.

15. De Latorre FJ, Klamburg J, Leon C, Soler M, Rius J: Incidence of Pneumothorax and Pneumomediastinum in Patients with Aspiration Pneumonia Requiring Ventilatory Support. Chest 1977, 72(2):141-144.

16. Gammon RB, Shin MS, Groves RH, Jr., Hardin JM, Hsu C, Buchalter SE: Clinical risk factors for pulmonary barotrauma: a multivariate analysis. Am J Respir Crit Care Med 1995, 152(4 Pt 1):12351240.

17. She WH, Chok KSH, Li IWS, Ma KW, Sin SL, Dai WC, Fung JYY, Lo CM: Pneumocystis jirovecii-related spontaneous pneumothorax, pneumomediastinum and subcutaneous emphysema in a liver transplant recipient: a case report. BMC Infect Dis 2019, 19(1):66-66.

18. Gammon RB, Shin MS, Buchalter SE: Pulmonary barotrauma in mechanical ventilation. Patterns and risk factors. Chest 1992, 102(2):568-572.

19. Weg JG, Anzueto A, Balk RA, Wiedemann HP, Pattishall EN, Schork MA, Wagner LA: The relation of pneumothorax and other air leaks to mortality in the acute respiratory distress syndrome. $N \mathrm{Eng} / \mathrm{J}$ Med 1998, 338(6):341-346.

20. Brochard L, Roudot-Thoraval F, Roupie E, Delclaux C, Chastre J, Fernandez-Mondéjar E, Clémenti E, Mancebo J, Factor $P$, Matamis $D$ et al: Tidal volume reduction for prevention of ventilator-induced lung injury in acute respiratory distress syndrome. The Multicenter Trail Group on Tidal Volume reduction in ARDS. Am J Respir Crit Care Med 1998, 158(6):1831-1838.

21. Hsu C-W, Sun S-F: latrogenic pneumothorax related to mechanical ventilation. World J Crit Care Med 2014, 3(1):8-14.

22. Petersen GW, Baier H: Incidence of pulmonary barotrauma in a medical ICU. Crit Care Med 1983, 11(2):67-69.

23. Miller MP, Sagy M: Pressure characteristics of mechanical ventilation and incidence of pneumothorax before and after the implementation of protective lung strategies in the management of pediatric patients with severe ARDS. Chest 2008, 134(5):969-973.

24. Brower RG, Ware LB, Berthiaume Y, Matthay MA: Treatment of ARDS. Chest 2001, 120(4):13471367.

25. Boussarsar M, Thierry G, Jaber S, Roudot-Thoraval F, Lemaire F, Brochard L: Relationship between ventilatory settings and barotrauma in the acute respiratory distress syndrome. Intensive Care Med 2002, 28(4):406-413. 
26. Chou CW, Lin FC, Tsai HC, Chang SC: The impact of concomitant pulmonary infection on immune dysregulation in Pneumocystis jirovecii pneumonia. BMC Pulm Med 2014, 14:182.

27. Kim T, Moon SM, Sung H, Kim MN, Kim SH, Choi SH, Jeong JY, Woo JH, Kim YS, Lee SO: Outcomes of non-HIV-infected patients with Pneumocystis pneumonia and concomitant pulmonary cytomegalovirus infection. Scand J Infect Dis 2012, 44(9):670-677.

28. Miles PR, Baughman RP, Linnemann CC, Jr.: Cytomegalovirus in the bronchoalveolar lavage fluid of patients with AIDS. Chest 1990, 97(5):1072-1076.

29. Korkmaz Ekren P, Töreyin ZN, Nahid P, Doskaya M, Caner A, Turgay N, Zeytinoglu A, Toz S, Bacakoglu F, Guruz $Y$ et al: The association between Cytomegalovirus co-infection with Pneumocystis pneumonia and mortality in immunocompromised non-HIV patients. The Clinical Respiratory Journal 2018, 12(11):2590-2597.

30. Töreyin ZN, Ekren P, Caner A, Töz S, Gürgün A, Uluer H, Bacakoglu F: Prognosis of < em > pneumocystis jirovecii</em > pneumonia in non-HIV patients. European Respiratory Journal 2013, 42(Suppl 57):P2759.

31. Choi SR: Changes in Cytomegalovirus Seroprevalence in Korea for 21 Years: a Single Center Study. 2018, 25(3):123-131.

32. Cook CH, Zhang Y, Sedmak DD, Martin LC, Jewell S, Ferguson RM: Pulmonary cytomegalovirus reactivation causes pathology in immunocompetent mice. Critical care medicine 2006, 34(3):842849.

33. Shaddock EJ, Richards GA, Murray J: Lung fibrosis in deceased HIV-infected patients with Pneumocystis pneumonia. 2012 2012, 13(2):4.

34. Zantah M, Dominguez Castillo E, Townsend R, Dikengil F, Criner GJ: Pneumothorax in COVID-19 disease incidence and clinical characteristics. Respiratory Research 2020, 21(1):236.

35. Roux A, Canet E, Valade S, Gangneux-Robert F, Hamane S, Lafabrie A, Maubon D, Debourgogne A, Le Gal S, Dalle F et al: Pneumocystis jirovecii pneumonia in patients with or without AIDS, France. Emerg Infect Dis 2014, 20(9):1490-1497.

36. Ingram RJ, Call S, Andrade A, White C, Wheeler D: Management and outcome of pneumothoraces in patients infected with human immunodeficiency virus. Clin Infect Dis 1996, 23(3):624-627.

37. Robert-Gangneux F, Belaz S, Revest M, Tattevin P, Jouneau S, Decaux O, Chevrier S, Le Tulzo Y, Gangneux JP: Diagnosis of Pneumocystis jirovecii pneumonia in immunocompromised patients by real-time PCR: a 4-year prospective study. J Clin Microbiol 2014, 52(9):3370-3376.

\section{Figures}




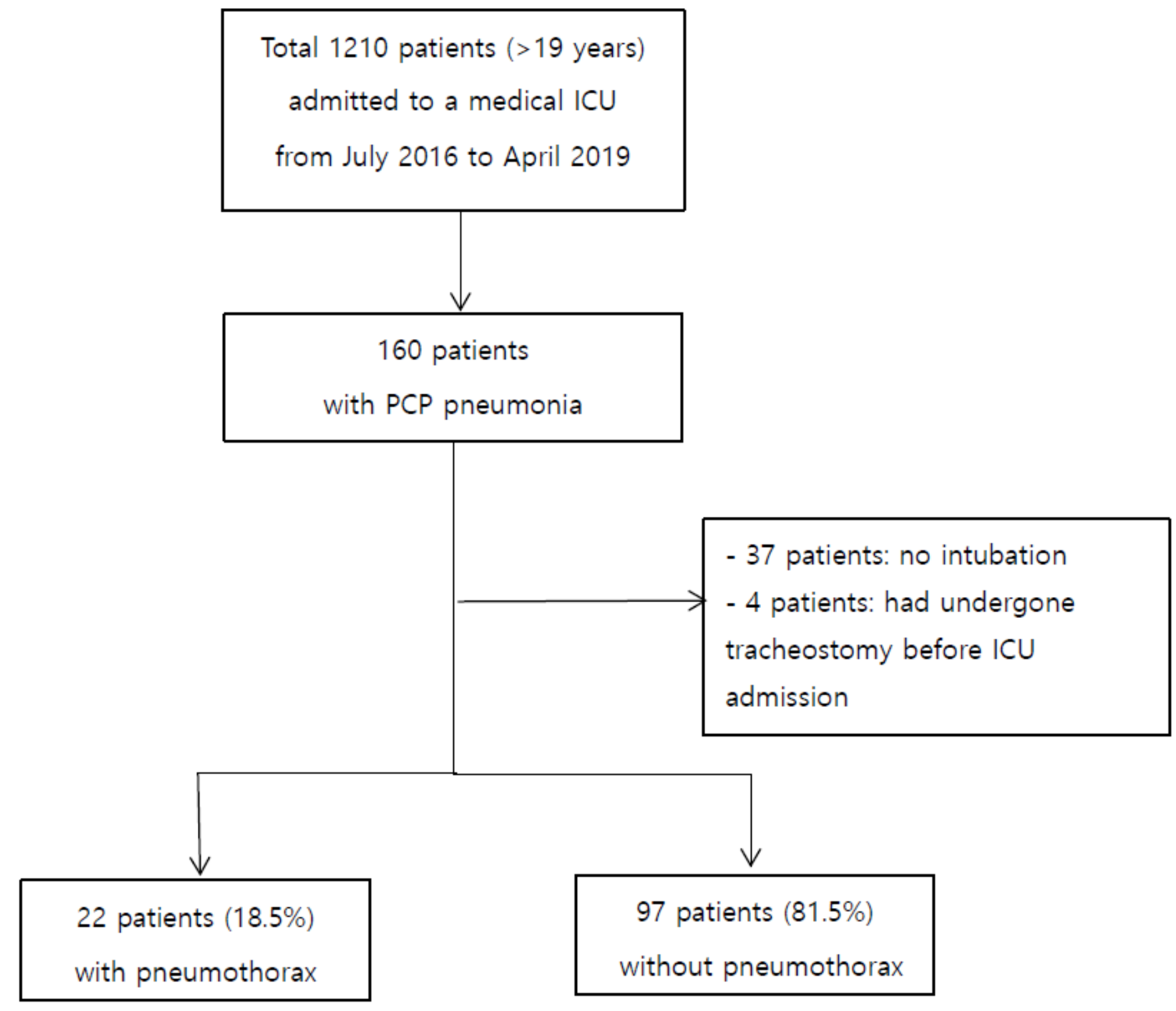

Figure 1

Flow diagram representing patient enrollment ICU, intensive care unit, PCP, Pneumocystis jirovecci pneumonia 